\title{
Magneto-Microfluidic Three-Dimensional Focusing of Magnetic Particles
}

\author{
Rana Afshar*, Yves Moser, Thomas Lehnert, and Martinus Gijs \\ Laboratory of Microsystems, Ecole Polytechnique Fédérale de Lausanne, 1015 Lausanne, Switzerland \\ *E-mail: rana.afshar@epfl.ch
}

\begin{abstract}
We present a method for magneto-microfluidic three-dimensional focusing of magnetic microparticles from a dense plug into a single streamline with longitudinal inter-particle spacing. Plug formation on-chip is induced by a highgradient magnetic field generated at the sidewall of a microchannel by an integrated micromachined magnetic tip that is connected to an external electromagnet. Controlled release of the microparticles is achieved using an exponential damping protocol of the magnetic retention force in the presence of an applied flow.
\end{abstract}

Keywords: Magnetophoresis, microfluidics, focusing, magnetic beads

PACS: $85.85 .+\mathrm{j}$

\section{INTRODUCTION}

Magnetic beads are versatile mobile carriers in microfluidics. The possibility to combine magnetic and fluidic forces for bead manipulation in small liquid volumes, for the extraction of target molecules from complex sample matrixes and for bead separation according to their size opens the way to innovative concepts with previously unmatched performance [1-3]. For accurate bead discrimination and counting, it is particularly attractive to implement on-chip an approach based on individual bead analysis, similar to cytometric techniques. Flow cytometry, however, requires 3-dimensional (3D) focusing of the particle suspension for reliable detection. Particles should pass one-by-one in-line and with a constant passage time through the illumination spot of the laser light coupled into the microchannel or precisely in front of an integrated detector [4-7]. 3D hydrodynamic focusing in a microfluidic device may be realized in a variety of ways, as was summarized in a recent review [8]. Both horizontal and vertical laminar sheath flow focusing concepts have been proposed, requiring a multilayer chip design with several fluidic inlets and eventually with different microchannel heights [9-10]. Dean flow has been used to perform the focusing [11-12]. (Di-)electrophoretic forces or acoustophoresis are other interesting options for focusing particles, cells or single molecules [13]. Magnetic force-based 3D focusing of magnetic beads in the centre of a microchannel has not been demonstrated so far.

$$
\vec{F}_{m a g}=\frac{1}{2 \mu_{0}} \Delta \chi V_{m} \nabla \vec{B}^{2}
$$

We present here an integrated microfluidic device, which allows 3D focusing of magnetic particles by combining magnetic and microfluidic forces. Magnetic field concentrators have been presented earlier in literature to generate high field gradients and magnetic forces, among which integrated microstructures have proven to be most efficient [14-15]. We use magnetophoresis to attract particles towards a magnetic tip prior to controlled release in a fluid flow. For the design of our system, the magnetic force $F_{\text {mag }}$ on the beads in a magnetic field $B$ was calculated by 
where $\Delta \chi$ is the magnetic susceptibility of the particle relative to the suspending medium, $\mu_{0}$ is the vacuum permeability and $V_{m}$ is the magnetically active volume of the particle [16-17]. This expression holds for unsaturated particles and is a good approximation for the present experiments.

\section{EXPERIMENTAL}

As is schematically shown in Fig. 1a, the system comprises a magnetic and a microfluidic part which are integrated on a single support and chip holder. These distinct parts are described in the following.

\section{Microfluidic Layout}

The microfluidic chip itself comprises a principal microfluidic channel (straight, $10 \mathrm{~mm}$ long, $200 \mu \mathrm{m}$ wide, 100 $\mu \mathrm{m}$ high) with a section for magnetic retention and a secondary microchannel (200 $\mu \mathrm{m}$ wide, $100 \mu \mathrm{m}$ high) that intercepts the main channel. Channels are cast in a single piece of poly-dimethylsiloxane (PDMS) using a standard SU8/Si molding technique [18]. Sealing of the channels is achieved by clamping the PDMS part onto a glass slide by means of a PMMA chip holder with suitable fluidic connections.

The fluidic junction of both channels is located $0.5 \mathrm{~mm}$ after the retention section (Fig. 1b). A single high precision syringe pump (neMESYS, Cetoni, Germany) is connected simultaneously to the fluidic inlets of both channels thus providing exactly the same flow rate of $0.3 \mathrm{~mm} / \mathrm{s}$ in both channel sections before the fluidic junction.

\section{Magnetic Setup}

The magnetic part of the system consists of an external coil, a magnetic yoke (PERMENORM) and a pair of soft magnetic tips (Fig. 1a). The magnetic microtips are cut by laser out of a $100 \mu \mathrm{m}$ thick magnetic foil (Vacoflux ${ }^{\circledR} 50$, Vacuumschmelze, Germany). The width of the narrow tip end is $50 \mu \mathrm{m}$. Magnetization of the tips is achieved by connecting their large end to the magnetic yoke. The PDMS part of the chip comprises recesses to receive the pair of magnetic poles in a reliable manner. Both tips are positioned asymmetrically with respect to the channel at a distance of $40 \mu \mathrm{m}$ and $500 \mu \mathrm{m}$ from the channel side wall, respectively (Fig. 1b). This configuration results in focusing and concentration of the externally generated magnetic field close to one channel wall. We use beads with a diameter of $1.0 \mu \mathrm{m}$ or $2.8 \mu \mathrm{m}$ in our experiments.
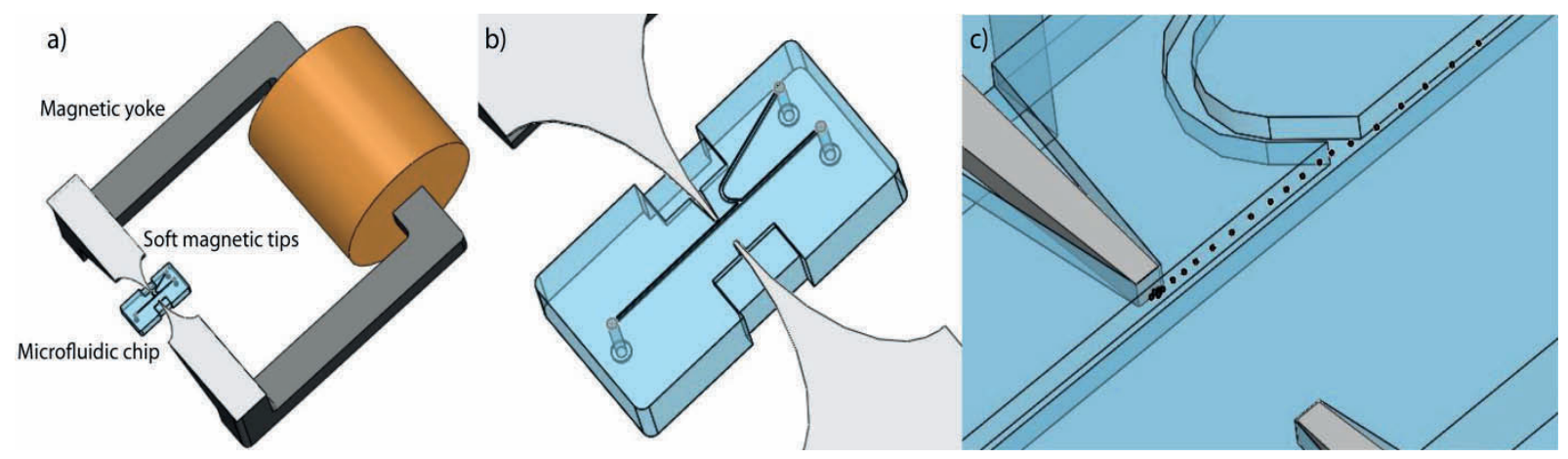

FIGURE 1. a) Electromagnetic bead actuation system comprising a coil, a magnetic yoke and a pair of soft-magnetic tips interfacing with the microfluidic chip; b) Microchip comprising a straight microfluidic channel, a curved secondary channel and the asymmetric arrangement of two magnetic poles (not to scale); c) Enlarged view of the zone of interest on the chip, showing schematically the release of a magnetic bead plug and the deviation of individual beads to form a 3D focused stream in the middle of the main channel. The width of the tip is $50 \mu \mathrm{m}$ and the channel width is $100 \mu \mathrm{m}$. 


\section{PROTOCOLS}

Two types of superparamagnetic beads with a diameter of $1.05 \mu \mathrm{m}\left(\Delta \chi=1.38\right.$, Dynabeads ${ }^{\circledR}$ MyOne $\left.^{\mathrm{TM}}\right)$ and 2.83 $\mu \mathrm{m}\left(\Delta \chi=0.84\right.$ Dynabeads $\left.{ }^{\circledR} \mathrm{M}-270\right)$, respectively, are used in the experiments. Bead solutions are diluted and suspended in $15 \mathrm{mM}$ phosphate buffer saline (PBS) solution with $0.5 \%$ surfactant (Tween 20) to improve the colloidal stability.

The first step of the experimental procedure is the capture of a well-defined amount of magnetic particles from the bead suspension which is introduced into the main channel. The captured beads form a highly localized plug on the channel side wall close to the adjacent tip (dosing, Fig. 2a). The surplus of bead solution is removed. Details of the corresponding protocol and characterization of dosing have been discussed previously by our group [19].

The second step is the controlled release of the plug and the formation of a confined stream of individual particles. This is achieved by exponential attenuation of the magnetic field, i.e. of the current in the coil and simultaneous application of a flow through the channel. A photograph of the plug release is shown in Fig. $2 \mathrm{~b}$. Superparamagnetic beads progressively lose their magnetic moment during attenuation of the magnetic field thus hydrodynamic shear forces increasingly counterbalance magnetic retention forces and tear beads away from the plug surface. Released particles are confined in a narrow stream within a distance of less than $20 \mu \mathrm{m}$ from the channel wall in the y-direction. The enlarged view of the region of interest in Fig. 1c illustrates schematically the release of a magnetically retained bead plug. The purpose of the incoming secondary flow is to deviate the particle stream towards the center of the main channel.

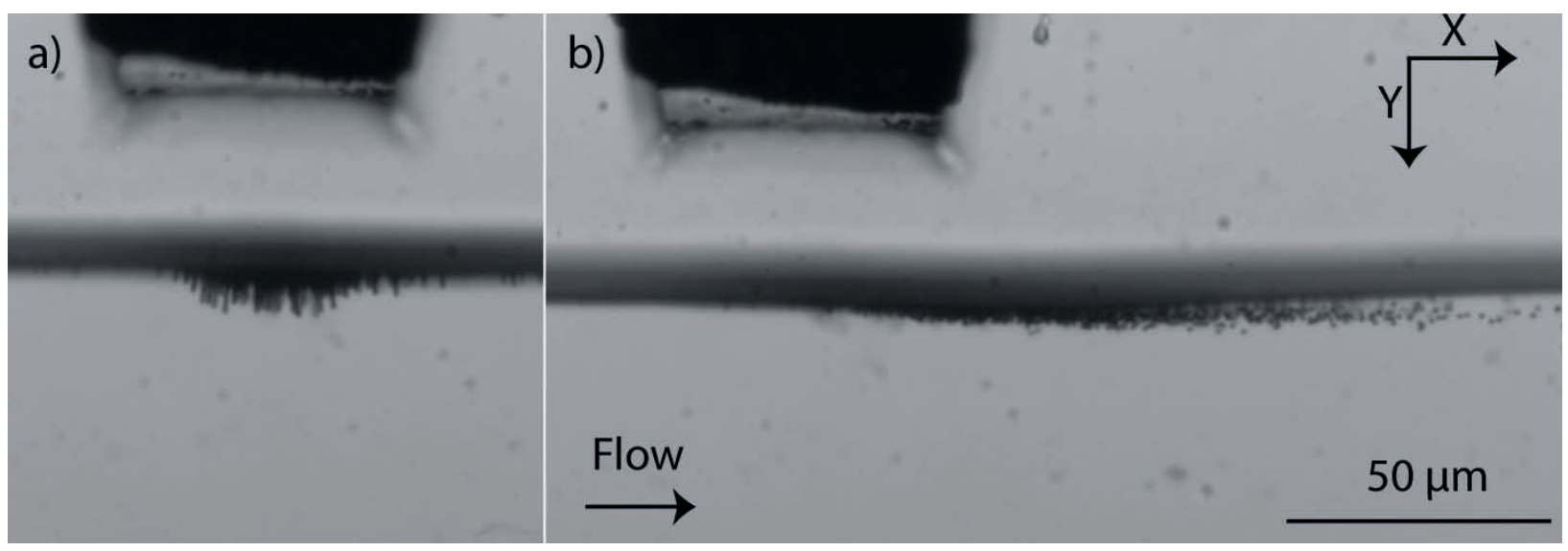

FIGURE 2. a) Top view (photograph) of a magnetic plug immobilized on the sidewall of the microchannel under the effect of an applied magnetic field. The plug consists of about 400 beads with a diameter of $1 \mu \mathrm{m}$; b) Beads are progressively released from the plug into the solution flow by controlled attenuation of the sinusoidal coil current that generates the magnetic retention field.

\section{RESULTS AND DISCUSSION}

Bead capture and plug formation near the magnetic tip on the channel wall, as well as subsequent release was demonstrated in the images shown in Fig. 2a and Fig. 2b, respectively. In the present case, parameters have been chosen to form a plug that contains $400 \pm 20$ beads of $1 \mu \mathrm{m}$ in diameter by using a coil current of $80 \mathrm{~mA}$. The asymmetric configuration of the magnetic field in y direction with respect to the channel middle axis attracts the beads towards the tip that is closest to the channel side wall.

The released stream of beads passes the fluidic junction on the chip, where it is deviated towards the center of the main channel (see Fig. 3). As both channel portions before the fluidic junction on the chip have equal hydraulic resistance, the flow rates in both sections are equal and as a result, the stream of the beads is deviated exactly to the middle of the main channel. After the junction, the flow rate and the maximum flow velocity are twice as high as in the upstream channel sections. Furthermore, beads are pushed from a region with very low flow velocity close to the channel wall towards the center of the parabolic flow profile. Particles in the released stream are therefore strongly 
accelerated. This experimental observation using $1 \mu \mathrm{m}$ beads is clear from the photograph of Fig. 3 . The final bead velocity in the main channel after the fluidic junction is $0.6 \mathrm{~mm} / \mathrm{s}$.

This simple fluidic arrangement allows stretching and one-by-one alignment of the released beads in the flow direction. Likewise, the lateral dimension of the focused stream varies. Reducing the maximum speed in the main channel from $0.6 \mathrm{~mm} / \mathrm{s}$ to $0.3 \mathrm{~mm} / \mathrm{s}$, for instance, resulted in a focused but $30 \mu \mathrm{m}$ wide particle stream with a dense distribution of particles. This is demonstrated by the photograph shown in Fig. 4. The velocity distribution of particles in the focused beam is very narrow.

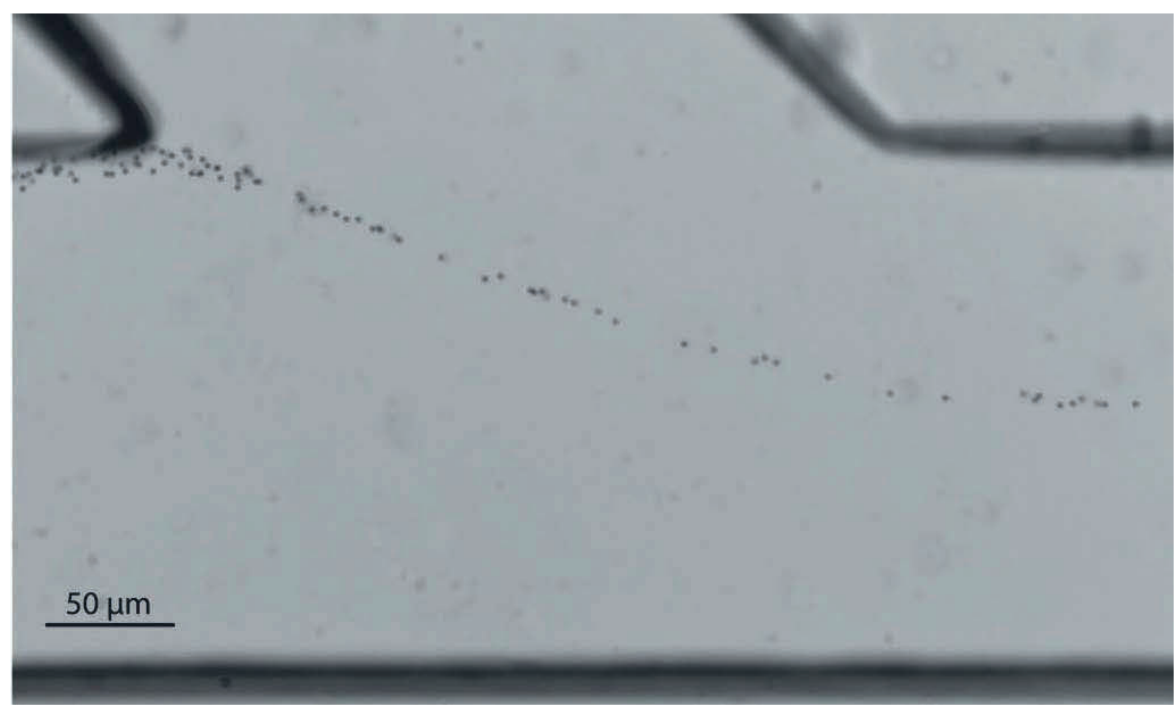

FIGURE 3: Photograph demonstrating focusing and alignment of $1 \mu \mathrm{m}$ beads during deviation and acceleration of the stream at the junction of the main and the secondary flow channels. The final bead velocity is $0.6 \mathrm{~mm} / \mathrm{s}$.

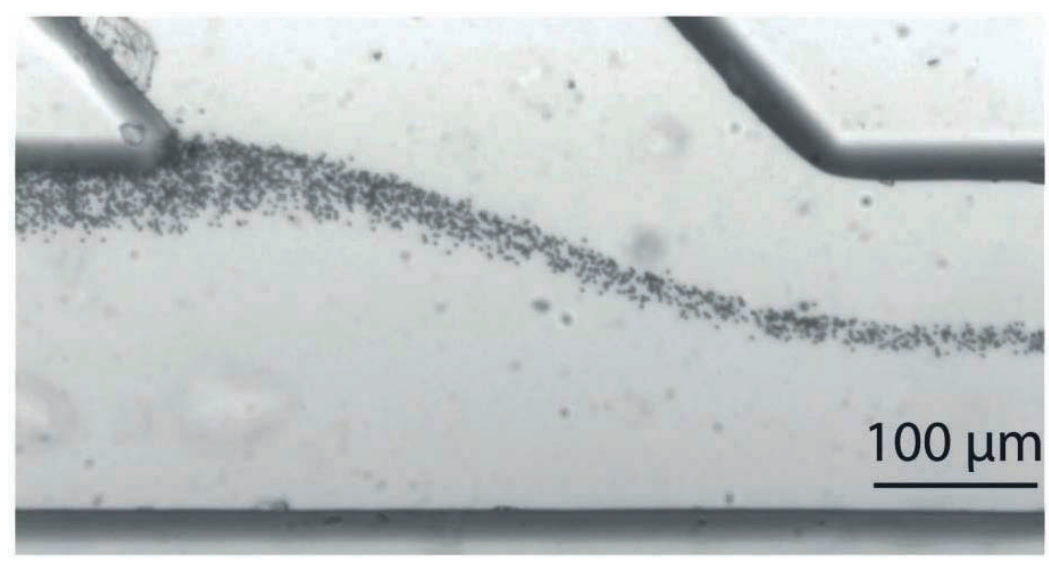

FIGURE 4: The lateral dimension of the focused stream varies as a function of the flow velocity. Reducing the maximum speed in the main channel to $0.3 \mathrm{~mm} / \mathrm{s}$ clearly results in a less confined bead stream (in this picture the number of beads in the plug is more than 400).

The out-of-plane (z-direction) field configuration near the tip is symmetric with respect to the channel height. Beads are therefore attracted towards half-height of the tip and the plug forms around this position. Experimental observation by confocal imaging confirmed this assumption. As a consequence, the released plug is readily focused in the z-direction at half the channel height. Confocal microscope imaging in the $\mathrm{x}-\mathrm{z}$ plane shows that the particles are focused within $10 \%$ of the height and width of the channel. A typical image of the channel cross section and the confined bead stream is shown in Fig. 5. The autofluorescent signal of the beads was recorded. Larger beads 
(diameter $2.8 \mu \mathrm{m}$ ) had to be used for this purpose in order to obtain a sufficiently high optical signal. At the location of the channel considered in Fig. 5, the beads appear not to be exactly focused at half height. Indeed, the center of the beam is deviated by $10 \mu \mathrm{m}$ from half-height of the channel towards the bottom. A quantitative analysis revealed that this deviation is due to sedimentation by the gravitational force acting on the beads after release. This effect is expected to be less pronounced with $1 \mu \mathrm{m}$ beads. The image shows a cross-section of the beam in the microchannel in the y-z plane taken $50 \mu \mathrm{m}$ after the fluidic junction. The flow speed in the center of the main channel is $0.6 \mathrm{~mm} / \mathrm{s}$. The focused beam diameter is about $10 \mu \mathrm{m}$ in z-direction and $15 \mu \mathrm{m}$ in y-direction. Considering the parabolic flow profile, the maximum variation of the bead velocity in the focused stream can be estimated to be about $1 \%$.

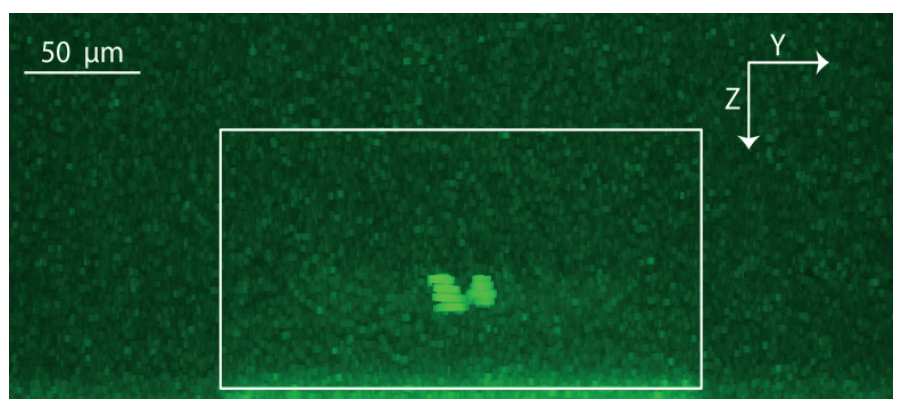

FIGURE 5: Cross-section of a focused $2.8 \mu \mathrm{m}$ bead stream in the main channel (rectangle) taken $50 \mu \mathrm{m}$ after the fluidic junction. The image is reconstructed from a series of fluorescent confocal images taken in the $\mathrm{x}-\mathrm{y}$ plane (vertical sectioning 3 $\mu \mathrm{m})$. The bead stream diameter is about $10 \mu \mathrm{m}$ in z-direction and $15 \mu \mathrm{m}$ in $\mathrm{x}$-direction. Using a bead velocity of $0.6 \mathrm{~mm} / \mathrm{s}$, the stream position is close to the center of the main channel cross-section.

Most of the microfluidic 3D focusing and on-chip flow cytometric systems proposed in literature target medium or high-throughput in order to meet specifications that approach the performance of commercial cytometers [20]. Often a compromise between accuracy of focusing, throughput and complexity of the design has to be found [4]. Unlike precedent approaches, the primary purpose of the system discussed in this paper is to provide a concept for manipulating a small and well-defined amount of functionalized magnetic beads. The integration of small magnetic microtips on-chip allows generating high gradients and adjustment of magnetic forces on the micrometer-sized particle with high accuracy. The present magneto-microfluidic 3D-focusing method requires only a single sheath flow and fluid control by one pump. This eliminates more complex hydrodynamic systems that use several sheath flows that are often difficult to operate. The device is fully compatible with regular microscopes and could be implemented as a low cost and simple alternative for particle focusing in biological applications.

\section{CONCLUSIONS AND OUTLOOK}

We presented a new method for 3D focusing of magnetic microparticles based on the interplay of magnetic and hydrodynamic forces. The asymmetry of the magnetic field configuration in the plane of the microfluidic channel allows for lateral confinement of the beads and subsequent controlled release in the flow. Deviation into the centre of the channel using a single sheath flow accelerates the beads resulting in a one-by-one alignment with constant particle velocity. Focusing in the vertical direction is readily achieved by taking advantage of the symmetry of a strongly focused magnetic field with respect to the channel height. As a consequence, very simple and robust particle focusing was obtained. Accuracy and stability of focusing appears to be compatible with the specifications of on-chip particle counting applications such as flow cytometry.

\section{ACKNOWLEDGMENTS}

The authors would like to thank the BioImaging and Optics Platform (BIOP) of EPFL (Lausanne), in particular Thierry Laroche for assistance in confocal imaging. The present work was supported by the European Commission funded project DETECTHIV (Grant No. 037118, Sensitive nanoparticle assay for the detection of HIV). 


\section{REFERENCES}

1. M. A. M. Gijs, F. Lacharme and U. Lehmann, Chemical Reviews 110 (3), 1518-1563 (2010).

2. M. A. M. Gijs, Microfluidics and Nanofluidics 1 (1), 22-40 (2004).

3. $\quad$ N. Pamme, Lab on a Chip 6 (1), 24-38 (2006).

4. C. Simonnet and A. Groisman, Analytical Chemistry 78 (16), 5653-5663 (2006).

5. X. Mao, S. C. S. Lin, C. Dong and T. J. Huang, Lab on a Chip 9 (11), 1583-1589 (2009).

6. $\quad$ N. Watkins, B. M. Venkatesan, M. Toner, W. Rodriguez and R. Bashir, Lab on a Chip 9 (22), 3177-3184 (2009).

7. E. P. Dupont, E. Labonne, C. Vandevyver, U. Lehmann, E. Charbon and M. A. M. Gijs, Analytical Chemistry 82 (1), 49-52 (2010).

8. X. Xuan, J. Zhu and C. Church, Microfluidics and Nanofluidics 9 (1), 1-16 (2010).

9. N. Sundararajan, M. S. Pio, L. P. Lee and A. A. Berlin, Journal of Microelectromechanical Systems 13 (4), $559-567$ (2004).

10. C. H. Tsai, H. H. Hou and L. M. Fu, Microfluidics and Nanofluidics 5 (6), 827-836 (2008).

11. X. Mao, J. R. Waldeisen and T. J. Huang, Lab on a Chip 7 (10), 1260-1262 (2007).

12. D. Di Carlo, Lab on a Chip 9 (21), 3038-3046 (2009).

13. J. Shi, X. Mao, D. Ahmed, A. Colletti and T. J. Huang, Lab on a Chip 8 (2), 221-223 (2008).

14. Y. Moser, T. Lehnert and M. A. M. Gijs, Applied Physics Letters 94 (2), 022505 (2009).

15. M. Zborowski, L. R. Moore, P. S. Williams and J. J. Chalmers, Separation Science and Technology 37 (16), 3611 - 3633 (2002).

16. M. Zborowski, L. Sun, L. R. Moore, P. Stephen Williams and J. J. Chalmers, Journal of Magnetism and Magnetic Materials 194 (1-3), 224-230 (1999).

17. S. S. Shevkoplyas, A. C. Siegel, R. M. Westervelt, M. G. Prentiss and G. M. Whitesides, Lab on a Chip 7 (10), 1294-1302 (2007).

18. S. K. Sia and G. M. Whitesides, Electrophoresis 24 (21), 3563-3576 (2003).

19. R. Afshar, Y. Moser, T. Lehnert and M. A. M. Gijs, Sensors and Actuators B: Chemical, DOI: 10.1016/j.snb.2009.1008.1044 (2009).

20. J. P. Nolan and L. A. Sklar, Trends in Biotechnology 20 (1), 9-12 (2002). 